Purpose To understand national changes in the quality of primary care reported by families of children and to estimate the potential benefits of recent health insurance expansions to children's receipt of primary care.

Methods A comparison of three iterations of the National Survey of Children's Health from 2003 to 2012 for children ages 0-18 years on measures of primary care experience (access, continuity, comprehensiveness and coordination). Trend analyses are stratified by insurance status and socio-demographics in order to assess the potential contribution of expanded coverage through health care reform to primary care experiences.

Results The results show very little change overall in primary care experiences for children nationally. Some significant gains were made in access and continuity for children considered more vulnerable (i.e., from families with lower-incomes and lower educational attainment), but were mostly explained by changes in insurance coverage over time.

Conclusion Insurance in the US remains a major factor in improving the primary care experience of children. The recent health care reforms have the potential to improve children's primary care access and continuity, but even with the intended goal of near universal insurance coverage for children, the US is not likely to reach the levels of paediatric primary care experience reported among studies in major European economies.

\section{0-194 INFANT AND YOUNG CHILD FEEDING PRACTICES IN UNDER 5 CHILDREN: CROSS-SECTIONAL SURVEY IN AN OUT PATIENT CLINIC}

'DV Patel, ${ }^{1}$ SC Bansal, ${ }^{1}$ AS Nimbalkar, ${ }^{2}$ AG Phatak, ${ }^{1}$ SM Nimbalkar. 'Department of Pediatrics, Pramukhswami Medical College, Karamsad, India; ${ }^{2}$ Central Research Services, Charutar Arogya Mandal, Karamsad, India

\subsection{6/archdischild-2014-307384.262}

Background and aims Infant and Young Child Feeding (IYCF) practices in under 5 children have great bearing on nutrition and health in childhood as well as in adult life. IYCF practices are influenced by local culture as well changes in education and health care interventions.

Methods Cross-sectional study. All mothers of children from 6 months to 5 years of age visiting Paediatric Outpatient Department were administered the questionnaire consisting of 39 questions relating to feeding practices and health of the child.

Results A total of 781 mothers were surveyed. More than half of the mothers $(57.5 \%)$ started feeding within an hour of birth, 67.2\% gave exclusive breastfeeding for six months, $51.6 \%$ continued breastfeeding for more than a year, $58.3 \%$ of the mothers breast fed 6-8 times, $24.6 \%$ more than 8 times and $17.1 \%$ less than 6 times, $30 \%$ of the mothers gave night feeds for 1-2 years, $18.2 \%$ of the mothers bottle fed the babies and $15.6 \%$ had problems during breastfeeding in first 6 months. $67.7 \%$ spend $<3 \mathrm{~h}$ daily on feeding the child. Multivariable logistic regression showed that early initiation of breastfeeding ( $p=0.037)$, hand hygiene $(p=0.03)$, food hygiene (storage: $\mathrm{p}=0.02$, Washing utensils: $\mathrm{p}=0.005$ ) and duration of exclusive breastfeeding $(\mathrm{p}<0.001)$ were significantly associated with hospitalisation of child. The predictive value of the model was fair with correct classification rate of $68.8 \%$.

Conclusions Current rates are well below the guidelines for IYCF feeding. Interventions to improve these practices need to be developed.

\section{Social Determinants of Health}

\section{0-195 MOTHERS' KNOWLEDGE AND PRACTICE ON MANAGEMENT OF CHILDHOOD ACUTE RESPIRATORY INFECTIONS IN ALBANIA}

'D Doracaj, 'E Grabocka, ${ }^{2} E$ Hallkaj. 'Basic Sciences, University of Medicine, Tirana, Albania; ${ }^{2}$ Faculty of Social Sciences, University of Tirana, Tirana, Albania

\subsection{6/archdischild-2014-307384.263}

Background and aims Acute respiratory infections (ARI) are the leading cause of death among children under five in Albania. This study aims to determine the parent's knowledge and care seeking practice related to ARI in children less than 2 years old in Albania.

Methods A cross-sectional household survey was conducted on June - July 2012 in the northeastern region of Albania. 600 mothers of children $0-23$ months selected using a multi-stage sampling technique were interviewed by trained health workers using a structured pre-tested questionnaire.

Results Knowledge: Only $8 \%$ of mothers recognised fast/difficult breathing as a danger sign of childhood illness requiring medical attention. $78 \%$ of interviewers were convinced that every child with fever had infection and $65 \%$ of them thought they need antibiotics.

Care seeking practice: Of the 116 children (19.3\%) who reported to have respiratory illnesses two weeks preceding the interview, $57.7 \%$ had an ARI. $70.2 \%$ of children with ARI were taken to a health facility and only $53.2 \%$ of them within the first 2 days. $63.8 \%$ of ARI cases sought medical care directly at the hospital bypassing the referral system. About $36.6 \%$ of cases seen by hospital providers were prescribed antibiotics compared to $11.7 \%$ of those seen by family doctors. $20 \%$ of mothers selfadministered antibiotics to their children, suggested by community pharmacists.

Conclusions Mothers' knowledge and care seeking practices related to ARI were poor. Community health education and strengthening of Integrated Management of Childhood Illnesses programme are required to improve timely and appropriate health care of childhood illnesses.

\section{0-196 ASSESSING EFFICIENT PATIENT CARE - SHOULD LENGTH OF STAY BE CALCULATED INDEPENDENTLY OF LOCAL ADMISSION RATES?}

${ }^{1}$ R Ross Russell, ${ }^{1}$ A Shahnaz, ${ }^{2} \mathrm{R}$ Parker, ${ }^{3} \mathrm{~S}$ Wills. ${ }^{1}$ Department of Paediatrics, Cambridge University Hospitals NHS Foundation Trust, Cambridge, UK; ${ }^{2}$ Medical Statistics, Centre for Applied Medical Statistics, Cambridge, UK; ${ }^{3}$ Information Management Department, Cambridge University Hospitals NHS Foundation Trust, Cambridge, UK

\subsection{6/archdischild-2014-307384.264}

Objective To compare the length of hospitalisation for infants with bronchiolitis across the Eastern region of the UK and to assess the impact of the varying admission rates in each hospital. Design Data collection through the Hospital Episode Statistics (HES) using the ICD clinical coding for bronchiolitis across all hospitals in East of England for three winter seasons (October to March for the years 2009/10, 2010/11 and 2011/12).

Main outcome measure: Length of hospital stay, corrected to adjust for local population.

Results Seventeen hospitals across the east of England were included in this study. Overall admission rate (as a percentage of 